\title{
Metástasis óseas, primera manifestación clínica de un carcinoma productor de calcitonina
}

\author{
M. Urdiales Viedma ${ }^{1}$, R. Carvia Ponsaille², J. Barranco García ${ }^{2}$, S. Martos Padilla ${ }^{1}$, R. López Urdiales ${ }^{1}$
}

\section{Resumen}

- Introducción: el carcinoma medular de tiroides comprende entre el 5\%-10\% de todos los carcinomas tiroideos. La presentación clínica más frecuente es una masa tiroidea.

- Caso clínico: varón de 32 años, con dolores debidos a múltiples metástasis óseas. Una biopsia de médula ósea mostró un carcinoma con positividad inmunohistoquímica a calcitonina y antígeno carcinoembrionario. Los niveles séricos de calcitonina y antígeno carcinoembrionario estaban muy altos. El paciente murió a los 12 meses del diagnóstico a pesar de ser sometido a quimio y radioterapia.

- Discusión: las metástasis a distancia son una manifestación inicial infrecuente en el carcinoma medular de tiroides. Pacientes con menos de 45 años tienen mejor supervivencia, pero la existencia de metástasis óseas se correlaciona con mal pronóstico. Las determinaciones inmunohistoquímicas y séricas de calcitonina y antígeno carcinoembrionario son importantes instrumentos diagnósticos.

- Conclusión: mostramos lo que creemos es un carcinoma medular de tiroides en un varón joven, con una presentación inicial atípica debido a múltiples metástasis óseas y curso fatal. La inmunohistoquimia es fundamental para diagnosticar el tumor primario en estas metástasis. En el caso de calcitonina y/o antígeno carcinoembrionario plasmático elevado y no explicado, es imprescindible descartar un carcinoma medular de tiroides.

\section{Palabras clave:}

Tiroides. Médula ósea. Carcinoma medular. Metástasis. Calcitonina. Antígeno carcinoembrionario.

Oncología, 2005; 28 (4):188-192

\footnotetext{
${ }^{1}$ Servicio de Anatomía Patológica

Hospital San Juan de la Cruz (Ubeda)

${ }^{2}$ Servicio de Anatomía Patológica

Hospital S. Agustín (Linares)

Jaén
} 


\section{Summary}

- Purpose: Thyroid medullary carcinoma comprises between 5 and $10 \%$ of all thyroid cancers. The most common clinical presentation is a thyroid mass. Study of a case with bone metastases.

- Case report: A 32 year old man with pain due to multiple bone metastases. A bone marrow biopsy disclosed a carcinoma immunologically positive to calcitonin and carcinoembryonic antigen. Serum level of both, calcitonin and carcinoembryonic antigen, were very high. The patient was treated with chemotherapy and radiotherapy, and died 12 months later.

- Discussion: Distant metastases are not a usual presentation of thyroid medullary carcinoma. Patients younger than 45 years have a better survival, but bone metastases correlate with a very bad prognosis. Immunohistochemistry and blood determination of carcinoembryonic antigen and calcitonin are important diagnostic tools.

- Conclusion: Atypical presentation of a thyroid medullary carcinoma in a young man with bone metastases and short survival. Immunohistochemistry is very useful to establish the origin of the primary tumour in these metastases. In the event of an unexplained plasma rise of calcitonin and/or carcinoembryonic antigen, it is mandatory to rule out a thyroid medullary carcinoma.

Key words: Thyroid. Bone marrow. Medullary carcinoma. Metastases. Calcitonin. Carcinoembryonic antigen.

\section{Introducción}

El carcinoma tiroideo es una neoplasia relativamente infrecuente, constituyendo entre el $0,5 \%$ $1,5 \%$ de todos los tumores malignos ${ }^{1}$. El carcinoma medular de tiroides (CMT), comprende sólo entre el 5-10\% de los cánceres tiroideos. La mayoría de los CMTs (70-80\%) son formas esporádicas, siendo los restantes (20-30\%) formas familiares, bien sin asociación a otros tumores o asociados a síndromes de neoplasias múltiples endocrinas (MEN 2A, MEN 2B $)^{2,3}$. En formas esporádicas del CMT, la presentación clínica más común es el hallazgo de un nódulo tiroideo ${ }^{4}$.

Presentamos un caso de un CMT en un varón joven, con una presentación clínica inicial rara, consistente en dolores óseos por múltiples metástasis óseas.

\section{Caso clínico}

Varón de 32 años, de edad, acude a urgencias del hospital, en febrero de 2000, por presentar dolor en hombro izquierdo y columna lumbar. El examen físico y analítica de rutina fue irrelevante. El estudio radiológico mostró lesiones óseas líticas generalizadas. TAC torácico abdominal no muestra imágenes sugerentes de neoplasia a nivel visceral. Gastroscopia, enema opaco, ecografía abdominal, testicular y prostática normales. A fin de establecer un diagnóstico, se practicó una biopsia de médula ósea, en cresta ilíaca. Microscópicamente, la médula ósea presentaba una sustitución parcial de su celularidad habitual, por una neoplasia constituida por nidos epiteliales, en un estroma fibroso (Fig. 1) en unas zonas, mientras en otras áreas dichos nidos están rodeados por la celularidad habitual de la médula ósea y están formados por células poligonales, con núcleos redondeados u ovales, de aspecto blando sin nucleolos prominentes y mitosis muy escasas. El estudio inmunohistoquímico mostró negatividad a receptores estrogénicos, proteína S-100, HMB45, p53, bc1-2 y sinaptofisina. Positividad en el $25 \%$ de los núcleos con MIB-1 y en el $90 \%$ de los núcleos con p27 y positividad difusa y fuerte a receptores de progesterona, cromogranina A, enolasa neuronal específica, antígeno carcinoembrionario y en menos 


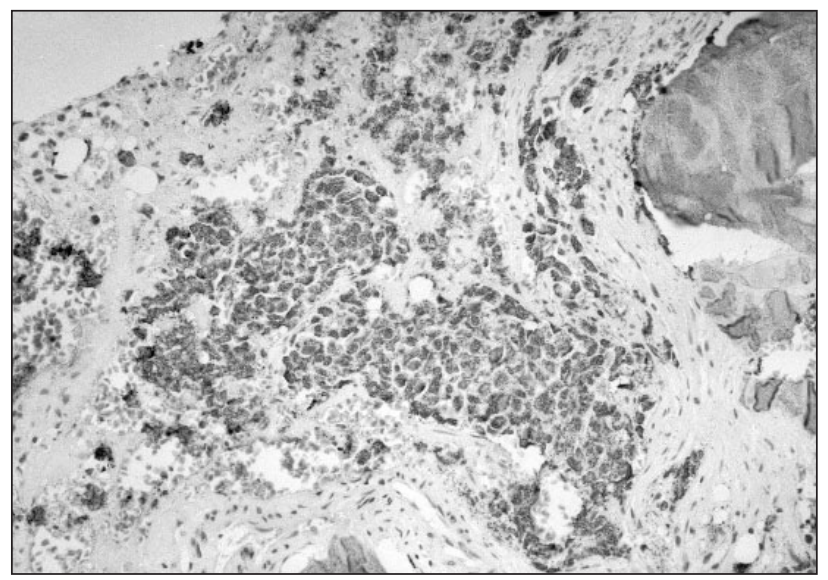

Figura 1. Médula ósea infiltrada por una neoplasia epitelial con estroma fibroso (HE, x 25).

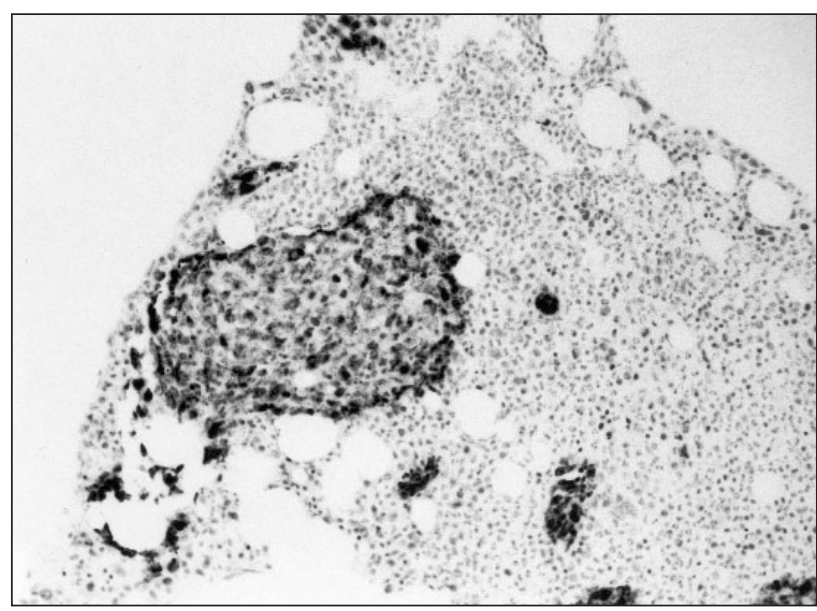

Figura 2. Nidos epiteliales en médula ósea, con positividad a calcitonina en $<50 \%$ de las células (x 25).

del 50\% de las células a calcitonina (Fig. 2), no se detectó amiloide con tinción de Rojo Congo. Con estos elementos se emitió un diagnóstico compatible con metástasis ósea de Carcinoma Medular de Tiroides. La determinación sérica de calcitonina fue de $3.500 \mathrm{pg} / \mathrm{ml}$ (normal de 15-100) y de CEA de 231 $\mathrm{ng} / \mathrm{ml}$ (normal de 0,01-5), siendo los niveles séricos de Ca 125, Ca 19.9 y PSA normales.

Dadas las malas condiciones físicas del paciente, éste fue enviado a Oncología de nuestro hospital de referencia en Jaén, donde se le encontró ecográficamente un pequeño nódulo tiroideo sólido, con calcio, en lóbulo izquierdo, que no se extirpa. Investigada la familia no se han encontrado antecedentes de patología tiroidea.
Se le administra una primera línea de quimioterapia (CDDP-ADR-VP16) durante 6 meses, sin respuesta. También se da radioterapia paliativa a la columna dorso lumbar. A principios de febrero de 2001, se inicia una segunda línea de quimioterapia, (CTX-DTIC), que se suspende a los pocos días por mal estado del paciente, progresión de metástasis a nivel óseo y aparición de metástasis pulmonar. El paciente fallece el 21 de febrero de 2001. La familia no autoriza la necropsia.

\section{Discusión}

Ocasionalmente se han descrito carcinomas neuroendocrinos principalmente en región gastroenteropancreática ${ }^{5,6}$ y de forma muy rara en otras localizaciones, como pueden ser la próstata o la laringe ${ }^{7,8}$, que han dado lugar a niveles séricos elevados de calcitonina.

Debido al rápido deterioro del paciente, no se le practicó una biopsia tiroidea.

Es interesante destacar que el paciente que presentamos tenía en la analítica inicial unos niveles altos de antígeno carcinoembrionario (ACE) y positividad tumoral con inmunohistoquimia a este antígeno. Aunque este marcador tiene poca especificidad y su elevación está estadísticamente más asociada a carcinomas de otras localizaciones (mama, tracto gastrointestinal). Ante subidas no explicadas de ACE se debe investigar un carcinoma medular de tiroides $^{9,10}$.

Con lo anteriormente expuesto, pensamos que al descartar mediante TAC, ecografía, enema opaco y endoscopia, la existencia de ninguna masa visceral, excepto el nódulo tiroideo junto a los altos niveles séricos de calcitonina y antígeno carcinoembrionario y finalmente la histología compatible de la metástasis ósea con carcinoma medular de tiroides, así como la comprobación por medio de la inmunohistoquímica, de la producción por parte de la neoplasia, de calcitonina y ACE creemos que en conjunto son datos que constituyen una base bastante sólida para pensar que estamos ante un carcinoma medular de tiroides metastático en hueso, aún cuando no dispongamos de confirmación histológica ni citológica del tumor primitivo.

Debido a la asociación del carcinoma medular de tiroides con otros tumores endocrinos ${ }^{2,3}$ y a su capa- 
cidad para producir diferentes hormonas ${ }^{2,4}$, la presentación clínica puede ser muy variada, con síndromes paraneoplásicos tan diferentes como diarrea severa $^{11}$, colostasis o hipercoagulabilidad sanguínea ${ }^{12}$. No obstante en casos de carcinoma medular de tiroides esporádicos (sin enfermedad familiar), la mayoría de los diagnósticos se establecen inicialmente al determinar una calcitonina en sangre tras descubrir un nódulo tiroideo ${ }^{4,13}$.

En caso de CMT, las metástasis mas frecuentes suelen ser en los linfáticos del cuello ${ }^{3}$, pero también se han descrito metástasis a pulmón, hígado, mama y con menor frecuencia y en estadios avanzados en huesos $^{3,14,15}$. La presentación de un CMT con dolores óseos como primer síntoma, debido a la existencia de metástasis óseas es muy raro. En la revisión que hemos realizado de la literatura de los últimos 10 años, en el Med-Line, únicamente hemos encontrado otro caso ${ }^{16}$ como el aquí presentado, en el que la primera manifestación de un carcinoma medular de tiroides sea la existencia de metástasis óseas en un paciente varón joven, y este es el primer caso sin nódulo palpable en tiroides.

La presencia de metástasis óseas se ha relacionado con un mal pronóstico, incluso peor que cuando existen metástasis distantes en vísceras o tejidos blandos ${ }^{15}$. De hecho este paciente se ha deteriorado rápidamente, razón por la que no se le practicó una tiroidectomía. Histológicamente, se ha descrito que cuando menos del $50 \%$ de las células tumorales son positivas a calcitonina, ese dato se asocia a peor pronóstico ${ }^{17}$, hecho que se da en el caso que presentamos. No obstante, debido a las numerosas metástasis óseas en este paciente en particular, posiblemente éstas tengan una incidencia mayor en su rápida y fatal evolución, que los hallazgos inmunobistoquímicos.

Finalmente, queremos llamar la atención sobre la utilidad de las técnicas inmunohistoquímicas, en la filiación de metástasis de origen desconocido. En este caso la positividad a calcitonina fue fundamental en el proceso diagnóstico.

\section{Bibliografía}

1. Danese F, Centanni M, Farsetti Andreali M. Diagnosis of thyroid carcinoma. J Exp Clin Cancer Res 1997; 16(3): $337-47$.

2. Katagiri M, Ontawa T, Ito K, Harada T. Familial non-multiple endocrine neoplasia medullary thyroid carcinoma: report of a case confirming a new clinical entity in Japan Surg -Today 1995; 25(9):822-6.

3. Rosai J, Carcangiu ML, De Lellis RAT. Medullary crcinoma in: Tumors of the thyroid gland. AFIP, Washington, D.C. 1992:207-45.

4. Ho C, Lin JD, Huang YY, Huang HS, Huang BY, Hsuen C. Clinical experience of medullary thyroid carcinoma in Chang Gung memorial Hospital. Chang Keng I Asueh 1996; 19(2):142-8.

5. Ichimura T, Kondo S, Okushiba S, Morikawa T, Coto H. A calcitonin and vasoaction intestinal peptide-producing pancreatic andocrine tumor associated with the WDHA síndrome. Int J Gastrointest Cancer 2003; 33(2-3):99-102.

6. Mahens A, Hoedecke J, Minze R, Thomusch O, Schneyer U, Dralle H. Hypercalcitoninemia in a sporadic asymptomatic neuroendocrine tumor of the pancreatic tail. Dig Surg 2000; 17(5):522-4.

7. Sim SJ, Glassman AB, Ro JY, Lee JJ, Logothetis GJ, Liu FJ. Serum calcitonin in small cell carcinoma ofthe prostate. Ann Clin Lab Sci 1996; 26(6):487-95.

8. Morales C, Tomas Bezos J, Alvarez- Quiñones Sanz M, García Mantilla J, Carrera F. Calcitonin producing neuroendocrine carcinoma of the larynx: Atypical carcinoid tumor. Acta Otorrinolaringol Esp 1996; 47(4):333-5.

9. Martínez M, Vivally ML, Guillausean-Scholer C, et al. High carcinoembryonic antigen level following cancer surgery: another way to detect thyroid medullary carcinoma. Rev Med Interne 2000; 21(10):897-900.

10. Behr TM, Becker W. Increased blood CEA level as inicial manifestation of medullary thyroid carcinoma. Dish Med Wochenschr 1999; 124(10):303-4.

11. Suter WR, Fantin AC, Meyenberger C. Diarrhea as initial manifestation of medullary thyroid carcinoma. Disch Med Wochenschr 1998; 123(39):1134-8.

12. Tiede DJ, Tefferi A, Kochhar R, Thompson GB, Hay ID. Paraneoplastic cholestasis and hypercoagulability associated with medullary thyroid carcinoma. Resolution with tumor debulking. Cancer 1994; 73(3):702-5.

13. Guyetant S, Dupre F, Bigorgne JC, et al. Medullary thyroid microcarcinoma: A clinicopathologic retropective study of 38 patients with no prior familial disease. Hum Pathol 1999; 30:1-7.

14. Ali, SZ, Teichberg S, Attie JN, Susin M. Medullary thyroid carcinoma metastatic to breast masquerading as infiltrating lobular carcinoma. Ann Clin Lab Sci 1994; 24 (5): 441-7.

15. Girelly ME, Nacamulli D, Pelizzo MR, et al. Medullary thyroid carcinoma: clinical features and long-term followup of seventy-eight patients treated between 1969 and 1986. Thyroid 1998; 8(6):517-23. 
M. Urdiales Viedma y cols.

16. Guerra Laso JM, de la Peña-Bataller R. Metástasis óseas múltiples e invasión de médula ósea como manifestación inicial de carcinoma medular de tiroides. An Med Interna 1997; 14(5):263-4.
17. Franc B, Rosenberg-Bourjin M, Caillou B, et al. Medullary thyroid carcinoma: search for histological predictors of survival (109 proband cases analysis). Hum Pathol 1998; 29(10):1078-84.

Correspondencia:

Dr. M. Urdiales Viedma

Unidad de Anatomía Patológica

Hospital San Juan de la Cruz

E-23400 Ubeda (Jaén)

E-mail: mariano.urdiales.sspa@juntadeandulucia.es 